\title{
Digital twin-based Optimization on the basis of Grey Wolf Method. A Case Study on Motion Control Systems
}

\author{
Rodolfo Haber \\ Centre for Automation and Robotics \\ (UPM-CSIC) \\ Madrid, Spain \\ rodolfo.haber@car.upm-csic.es \\ Fernando Castaño \\ Centre for Automation and Robotics \\ (UPM-CSIC) \\ Madrid, Spain \\ fernando.castano@car.upm-csic.es
}

\author{
Stanisław Strzelczak \\ University of Technology \\ Warsaw, Poland \\ s.strzelczak@wip.pw.edu.pl
}

Luca Fumagalli

Department of Management,

Economics and Industrial Engineering,

Politecnico di Milano, Milano, Italy

luca1.fumagalli@polimi.it

\author{
Zoran Miljković \\ Department of Production Engineering \\ University of Belgrade, \\ Belgrade, Serbia \\ zmiljkovic@mas.bg.ac.rs \\ Milica Petrović \\ Department of Production Engineering \\ University of Belgrade, \\ Belgrade, Serbia \\ mmpetrovic@mas.bg.ac.rs
}

\begin{abstract}
Nowadays, digital twins are fostering the development of plug, simulate and optimize behavior in industrial cyber-physical systems. This paper presents a digital twin-based optimization of a motion system on the basis of a grey wolf optimization (GWO) method. The digital twin of the whole ultraprecision motion system with friction and backlash including a P-PI cascade controller is used as a basement to minimize the maximum position error. The simulation study and the real-time experiments in trajectory control are performed to compare the performance of the proposed GWO algorithm and the industrial method called Fine tune (FT) method. The simulation study shows that the digital twin -based optimization using GWO outperformed FT method with improvement of $66.4 \%$ in the reduction of the maximum position error. The real-time experimental results obtained show also the advantage of GWO method with $18 \%$ of improvement in the maximum peak error and $16 \%$ in accuracy.
\end{abstract}

Keywords-digital twin, optimization, grey wolf optimizer, controller tuning, CNC machine tools

\section{INTRODUCTION}

Nowadays, a set of modeling and control methods for Industrial cyber-physical systems (ICPS) is available to enable new cooperation and coordination levels for achieving better efficiency short time-to-market solutions and more flexibility [1-3]. Several gaps and research challenges in ICPS should be addressed in short terms $[4,5]$. Digital twins (DT) have emerged as a powerful strategy to deal with multiphysical, multiscale and heterogeneity of systems $[6,7]$. Key aspects of DTs in relation to big data applications and a pathway to integrate them according to Industry 4.0 paradigm to foster smart manufacturing solutions were carefully analyzed in [8]. Certainly, the use of big data tools and methods in ICPS to deal with product lifecycle is not straightforward. Some contemporary studies have shown how efficient methods can be designed and deployed to address this hot topic on the basis of a DT-based procedure [9]. New research working towards shop-floor DT systems was reported in the literature as well as key components ranging from physical to DT data [10]. Nowadays, dozens of scientific and technical reports have demonstrated the wide range of DT applications in ICPS such as design of sensoring systems [11, 12], system integration and diagnostics [13], smart prediction and promising web services [14]. Recently, swarm intelligence and gradient free optimization methods have received more attention due to better computational framework and more efficient computing resources [15-18]. Soft-computing and machine learning have also demonstrated high potential in ICPS [19-21], but still few solutions based on DT. One challenging issue is related with the accuracy and exploitability of DTs in smart manufacturing. Indeed, manufacturing data and sensory data can be integrated into DTs of virtual systems to improve the overall behavior of the physical system $[22,23]$. DTs in ICPS are lacking:

- clear engineering procedures for systematic application of Artificial Intelligence, mainly machine learning methods, for designing and implementing DTs,

- focus on feedback systems including human-in the-loop and closed-loop control system performance,

- procedures for considering real-time constraints and real time issues in AI-based DTs and,

- systematic procedures for DT-based optimization and adaptation procedures for smart manufacturing.

From the viewpoint of modeling and control, traditional and AI-based modeling and control strategies have been explored in laboratories [24-26] but technology transfer is still cumbersome [27]. Therefore, DT are essential to address the unsatisfactory behavior of industrial systems due to poor tuning of controllers. For example, the calibration of cascade control systems is a cumbersome task for nonlinear systems, specifically before hard nonlinearities [28]. In the last two decades, nature-inspired metaheuristic algorithms were designed and implemented to tackle complex optimization problems and to provide a set of quasi-optimal solutions [29]. The advantages of swarm intelligence motivated the use of these methods in order to improve the tuning of P-PI cascade controllers' parameters on the basis of DT. In this paper, a population-based metaheuristic method called grey wolf optimizer (GWO) is re-designed and tailored in a digital twin procedure shown in Fig. 1 to optimally set control and compensation parameters of motion system. The cost function or main figure of merit is maximum position error. Therefore, it is necessary to yield a set of parameters able to decrease as much as possible this error keeping the overall accuracy 
without remarkable increase in the control effort. The performance of the presented GWO method is evaluated by means of a simulation study and real-time experiments.

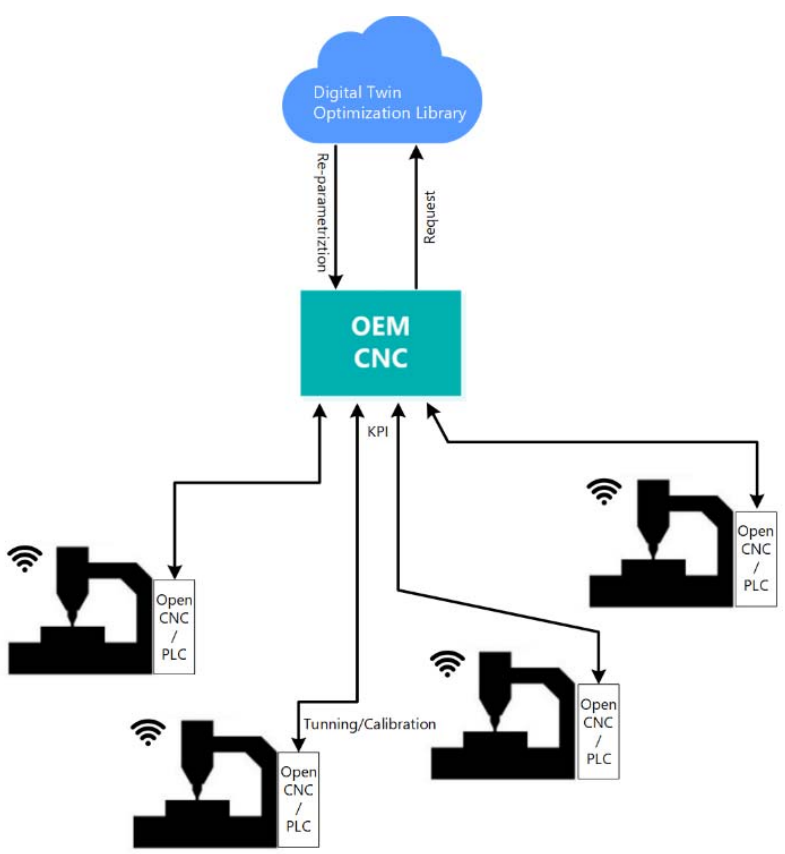

Fig. 1. Diagram of the DT-based Optimization approach

The structure of the paper is as follows. After a brief introductory overview of the physical and digital twins, Section 2 introduces the physical system and the corresponding digital twin. Section 3 presents a brief description of GWO algorithm, while Section 4 presents an industrial Fine Tune (FT) method applied for tuning of P-PI controller parameters. The simulation results and real-time experimental results are depicted in Section 5. The concluding remarks are summarized in Section 6.

\section{Digital Twin}

\section{A. Physical System Description}

The real case study is based on a DT of a real ultraprecision motion system commonly available in machine tools. Nowadays, open Computerized Numerical Control (oCNC) are a key element to guarantee not only accurate and fast manufacturing but also for enabling communication with other machines and devices. The experimental platform is shown in Fig. 2.

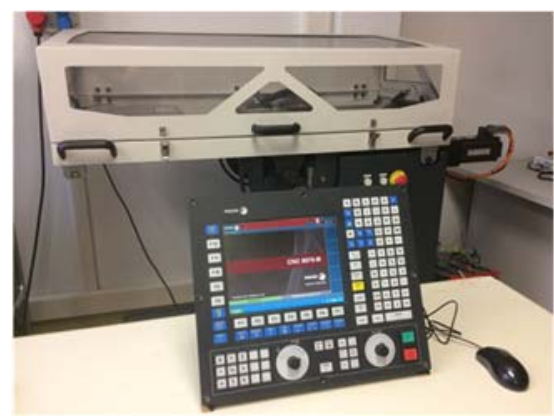

Fig. 2. Test platform

The regulation of position and velocity is performed by a P-PI cascade controller running in the open CNC. Real-time data, the controller' parameters and key variables can be easily gathered via open CNC. The most important parameters of the whole physical system are summarized in Table I.

TABLE I PHYSICAL SYSTEM

\begin{tabular}{|c|c|c|}
\hline Symbol & Quantity & Value \\
\hline$J_{\mathrm{M}}$ & Motor inertia & $2.9 \cdot 10^{-4} \mathrm{~kg} \cdot \mathrm{m}^{2}$ \\
\hline$J_{\mathrm{L}}$ & Load inertia & $4.2 \cdot 10^{-4} \mathrm{~kg} \cdot \mathrm{m}^{2}$ \\
\hline$f_{1}, \omega_{01}$ & Resonance frequency & $70 \mathrm{~Hz}, 2 \pi f_{1} \mathrm{rad} / \mathrm{s}$ \\
\hline$D_{1}$ & Damping coefficient & 0.15 \\
\hline$K_{\mathrm{t}}$ & Torque constant & $0.74 \mathrm{~N} \cdot \mathrm{m} / \mathrm{A}$ \\
\hline$f r_{\mathrm{H}}$ & Actual hysteresis & $0.1280 \mathrm{rad} / \mathrm{s}$ \\
\hline$F_{\mathrm{C}}$ & Coulomb friction & $0.625 \mathrm{~N} \cdot \mathrm{m}$ \\
\hline \multirow[t]{2}{*}{$F_{\mathrm{V}}$} & Viscous friction & $0 \mathrm{~N} \cdot \mathrm{m} \cdot \mathrm{s}$ \\
\hline & Backlash & $12.2 \cdot 10^{-6} \mathrm{~m}$ \\
\hline$K_{\mathrm{H}}$ & Spindle Constant & $0.010 /(2 \pi) \mathrm{m} / \mathrm{rad}$ \\
\hline NP121 & $\begin{array}{l}\text { Input revolutions for } \\
\text { coupling the motor shaft } \\
\text { and leadscrew }\end{array}$ & 3 \\
\hline NP122 & $\begin{array}{l}\text { Output revolutions, } \\
\text { NP121/NP122 is the } \\
\text { gear ratio }\end{array}$ & 2 \\
\hline NP123 & $\begin{array}{l}\text { Feed constant for linear } \\
\text { axis }\end{array}$ & $0.02 \mathrm{~m}$ \\
\hline NP1 & $\begin{array}{l}\text { Reduced actuated } \\
\text { momentum of inertia }\end{array}$ & $144.61 \%$ \\
\hline$T_{s 1}, T_{s 2}$ & $\begin{array}{l}\text { Sampling time for } \\
\text { position and velocity } \\
\text { loops }\end{array}$ & $250 \mu \mathrm{s}, 62.5 \mu \mathrm{s}$ \\
\hline
\end{tabular}

\section{B. Digital twin description.}

The DT is composed of two key virtual modules, one for representing the electromechanical system and another one for emulating the control systems. The two masses and spring system serves as basement for representing the motion system including the motor, the shaft, and the load. [26]. If the resonance and antiresonance frequencies, $\omega 01$ and $\omega 02$, are defined as:

$$
\begin{aligned}
& \omega_{01}=\sqrt{K / J_{\mathrm{L}}} \\
& \omega_{02}=\omega_{01} \sqrt{1+\left(J_{\mathrm{L}} / J_{\mathrm{M}}\right)} ;
\end{aligned}
$$

and, the damping coefficients, $D_{1}$ and $D_{2}$, are:

$$
\begin{aligned}
& D_{1}=\frac{B}{2 \omega_{01} J_{\mathrm{L}}} \\
& D_{2}=D_{1} \sqrt{1+\frac{J_{\mathrm{L}}}{J_{\mathrm{M}}}}=\frac{B\left(J_{\mathrm{M}}+J_{\mathrm{L}}\right)}{2 J_{\mathrm{M}} J_{\mathrm{L}} \omega_{02}}
\end{aligned}
$$

The transfer function that relates motor speed and torque is:

$$
H_{\omega_{\mathrm{M}} / M_{\mathrm{M}}}(s)=\frac{1}{J_{\mathrm{M}} s} \cdot \frac{s^{2}+2 D_{1} \omega_{01} s+\omega_{01}^{2}}{s^{2}+2 D_{2} \omega_{02} s+\omega_{02}^{2}}
$$

By using the previously defined values of, $\omega_{02}, D_{1}$, and $D_{2}$, the transfer function relating motor and load velocities becomes:

$$
H_{\omega_{\mathrm{L}} / \omega_{\mathrm{M}}}(s)=\frac{2 D_{1} \omega_{01} s+\omega_{01}^{2}}{s^{2}+2 D_{1} \omega_{01} s+\omega_{01}^{2}}
$$


Moreover, an electric model that relates electric current to the torque developed by the motor is also considered in the DT. Friction, backlash and noise are also considered in the DT, through computationally efficient models. Friction is represented by a viscous friction component, FV, relies on the relative velocity between the surfaces, $\mathrm{v}$, depicted in (5):

$$
F=F_{\mathrm{C}} \operatorname{sgn}(v)+F_{\mathrm{v}} v .
$$

The backlash compensation is also represented by virtual element. The backlash peak amplitude PP2 and peak time PP3 are essential for increasing motor speed during a time period making possible the exponential compensation due to movement reversal peak, can be:

$$
R_{P}=P P_{2} \mathrm{e}^{-t / P P_{3}}
$$

The sixth parameter is the hysteresis amplitude, $\mathrm{fH}$. The compensation of these non-linearities requires to face the zero cross discontinuity problem, solved by appropriately set of the hysteresis amplitude.

Therefore, on the basis of the relevance of the six parameters previously explained, the DT-based optimization method for an ultraprecision motion system sets the following parameter's vector:

$$
K=\left[\begin{array}{lll}
K_{\mathrm{p}}^{\mathrm{pos}} K_{\mathrm{p}}^{\mathrm{vel}} K_{i}^{\mathrm{vel}} P P_{2} P P_{3} f_{\mathrm{H}}
\end{array}\right]
$$

where, $K_{\mathrm{p}}^{\mathrm{pos}}$ is the proportional gain of the position controller; $K_{\mathrm{p}}^{\mathrm{vel}}, K_{i}^{\mathrm{vel}}$ are the proportional and integral gain, respectively, of the speed controller. $P P_{2}$ and $P P_{3}$ are the peak amplitude and peak time for compensating the backlash and, $f_{H}$ is the compensator for the friction hysteresis.

\section{GREY WOLF OPTIMIZATION ALGORITHM}

The Grey Wolf Optimization (GWO) algorithm, initially proposed by Mirjalili et al. in [30], belongs to class of novel swarm-based meta-heuristics inspired from the social leadership and hunting technique of grey wolves in nature. According to GWO algorithm, the grey wolves are classified into four levels of social hierarchy: alpha $(\alpha)$, beta $(\beta)$, delta $(\delta)$, and omega $(\omega)$. The alphas are the leaders of the group responsible for hunting process and making decisions. The betas belong to the second level of hierarchy and they assist the alphas in making decisions, while deltas belong to the third level and dominate the wolves of the last level omega. The omegas are the lowest ranking grey wolves on the pyramid of social hierarchy.

GWO algorithm is based on the aforementioned social behavior of the grey wolves. Optimization (hunting) process is initialized with randomly generated candidate solutions (grey wolves) in a multi-dimensional search space. This phase of searching for prey is also known as exploration. The best fitness solution is defined as alpha $(\alpha)$, the second and third best solutions are beta $(\beta)$ and delta $(\delta)$, and the rest of the solutions are assumed to be omega $(\omega)$. In order to catch the prey, the $\alpha, \beta$, and $\delta$ grey wolves firstly encircle the victim. During the optimization (hunting) process, they estimate the victim position and update their positions randomly around the victim according to the mathematical model given by the following equations (8) and (9):

$$
\begin{array}{r}
D=\left|\vec{C} \cdot \vec{X}_{p}(\mathrm{t})-\vec{X}(\mathrm{t})\right| \\
\vec{X}(\mathrm{t}+1)=\vec{X}_{p}(\mathrm{t})-\overrightarrow{\mathrm{A}} \cdot \overrightarrow{\mathrm{D}}
\end{array}
$$

where $t$ represents iteration, $\vec{X}_{p}$ is the position vector of the prey, $\vec{X}$ is the position vector of a grey wolf, $\vec{A}$ and $\vec{C}$ are coefficient vectors calculated by (10), (11):

$$
\begin{aligned}
& \vec{A}=2 \vec{a} \cdot \vec{r}_{1}-\vec{a} \\
& \vec{C}=2 \cdot \vec{r}_{2}
\end{aligned}
$$

The components of vector $\vec{a}$ linearly decrease from 2 to 0 , and $r_{1}$ and $r_{2}$ are random vectors in $[0,1]$.

Furthermore, hunting behavior of the grey wolves can be mathematically modeled by equations (12), (13) and (14):

$$
\begin{aligned}
& D_{\alpha}=\left|\vec{C}_{1} \cdot \vec{X}_{\alpha}-\vec{X}\right| \\
& D_{\beta}=\left|\vec{C}_{1} \cdot \vec{X}_{\beta}-\vec{X}\right| \\
& D_{\delta}=\left|\vec{C}_{1} \cdot \vec{X}_{\delta}-\vec{X}\right|
\end{aligned}
$$

The positions of $\alpha, \beta$, and $\delta$ grey wolves (the first free best solutions) are updated according to the following equations:

$$
\begin{gathered}
\vec{X}_{1}=\vec{X}_{\alpha}-\vec{A}_{1}\left(\overrightarrow{\mathrm{D}}_{\alpha}\right) \\
\vec{X}_{2}=\vec{X}_{\beta}-\vec{A}_{2}\left(\overrightarrow{\mathrm{D}}_{\beta}\right) \\
\vec{X}_{3}=\vec{X}_{\delta}-\vec{A}_{3}\left(\overrightarrow{\mathrm{D}}_{\delta}\right) \\
\vec{X}(\mathrm{t}+1)=\frac{\vec{X}_{1}+\vec{X}_{2}+\vec{X}_{3}}{3}
\end{gathered}
$$

Finally, the hunting process is finished by attaching the prey (exploitation phase). The pseudocode of GWO algorithm is shown in Table II.

\section{TABLE II. PSEUdO CODE OF GWO ALGORITHM}

Initialize the GWO algorithm (population size, maximum number of iterations, position vector $X$, and vectors $A, a, C$ );

Initialize a population of grey wolves (11)-(12);

Evaluate each grey wolf's fitness function by using (10);

Identify three best wolves (the best search agent - $X_{\alpha}$, the second best

search agent $-X_{\beta}$, the third best search agent $-X_{\delta}$ ) according to their

fitness functions;

Repeat

generate next population by updating each agent position (15)-(17); update $a, A, C$ by using (10) and (11);

compute each agent's fitness function (8); update $X_{\alpha}, X_{\beta}, X_{\delta}$;

Until the maximum of generation is not met

Output: the optimal parameters

$$
\left[K_{\mathrm{p}}^{\mathrm{pos}} K_{\mathrm{p}}^{\mathrm{vel}} K_{i}^{\mathrm{vel}} P_{2} P_{2} P_{3} f_{\mathrm{H}}\right]_{\mathrm{OPT}}
$$




\section{FINE TUNE METHOD}

The Fine Tune (FT) method is a well-known method to manually tune CNC machine tools in order to improve their performance. Different experimental-based procedures combined with computational intelligence methods such as expert systems or fuzzy logic, as well as information about knowledge accumulated in databases, can be used for fine tuning of one axis or all CNC axes automatically. Finally, the tuning of servo-performance of $\mathrm{CNC}$ machine tools axes is carried out by combining experimental studies and frequency response diagrams. More details about FT method can be found in [31].

\section{EXPERIMENTAL RESULTS}

\section{A. Simulation results}

This section provides information related to parameters setting of proposed algorithms. The implementation of the method is carried out Matlab R2019 software package. The personal computers has an Intel Core i7-4790 CPU $3.6 \mathrm{GHz}$ with 16GB RAM. The main parameters of the GWO are the size of population 20, the maximum number of iterations 100 , $a$ linearly decreasing of $a$ parameter $[2,0]$, and random vectors $[0,1]$ for $r_{l}$ and $r_{2}$.

The simulation results of GWO algorithm using the digital twin are compared with the results achieved by FT method. The optimal parameters are $\mathbf{K}_{\mathrm{FT}}=[66.67,0.29,0.008$ $0.718,0.008,0.1288]$ and $\mathbf{K}_{\mathrm{GWO}}=[75.00,0.2632,0.0012$ $0.4368,0.04393,0.00231]$. The average computing time for FT is $14.410^{3} \mathrm{~s}$ whereas for GWO it takes $9.75 \cdot 10^{3} \mathrm{~s}$. Fig. 3 shows the reference position and velocity used in simulation and experiments of the tracking and trajectory control in experiments that lasted 32.76 seconds. These reference values are further used for validating the proposed digital twin-based GWO methodology. Fig. 4 shows how GWO algorithm achieves the best performance index with a remarkable reduction in $\max E$ quantified in $66.4 \%$ in comparison with the Fine Tune method. The simulation results of position error are presented in Fig. 5, including a zoom in, and the control signal is presented in Fig. 5. The dynamic response shown in Fig. 5 corroborates the good accuracy of the GWO for tracking the desired trajectory.

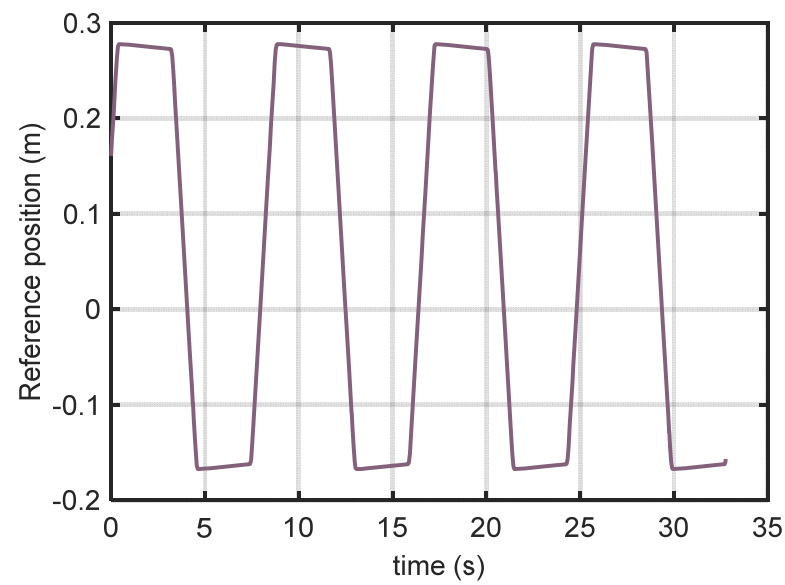

Fig. 3 The reference position for trajectory control.

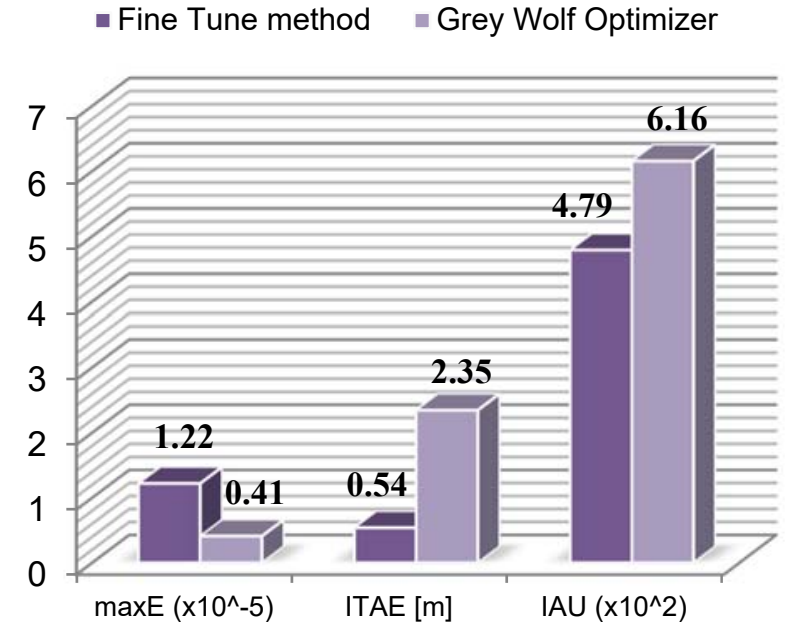

Fig. 4 Performance indices in simulation results
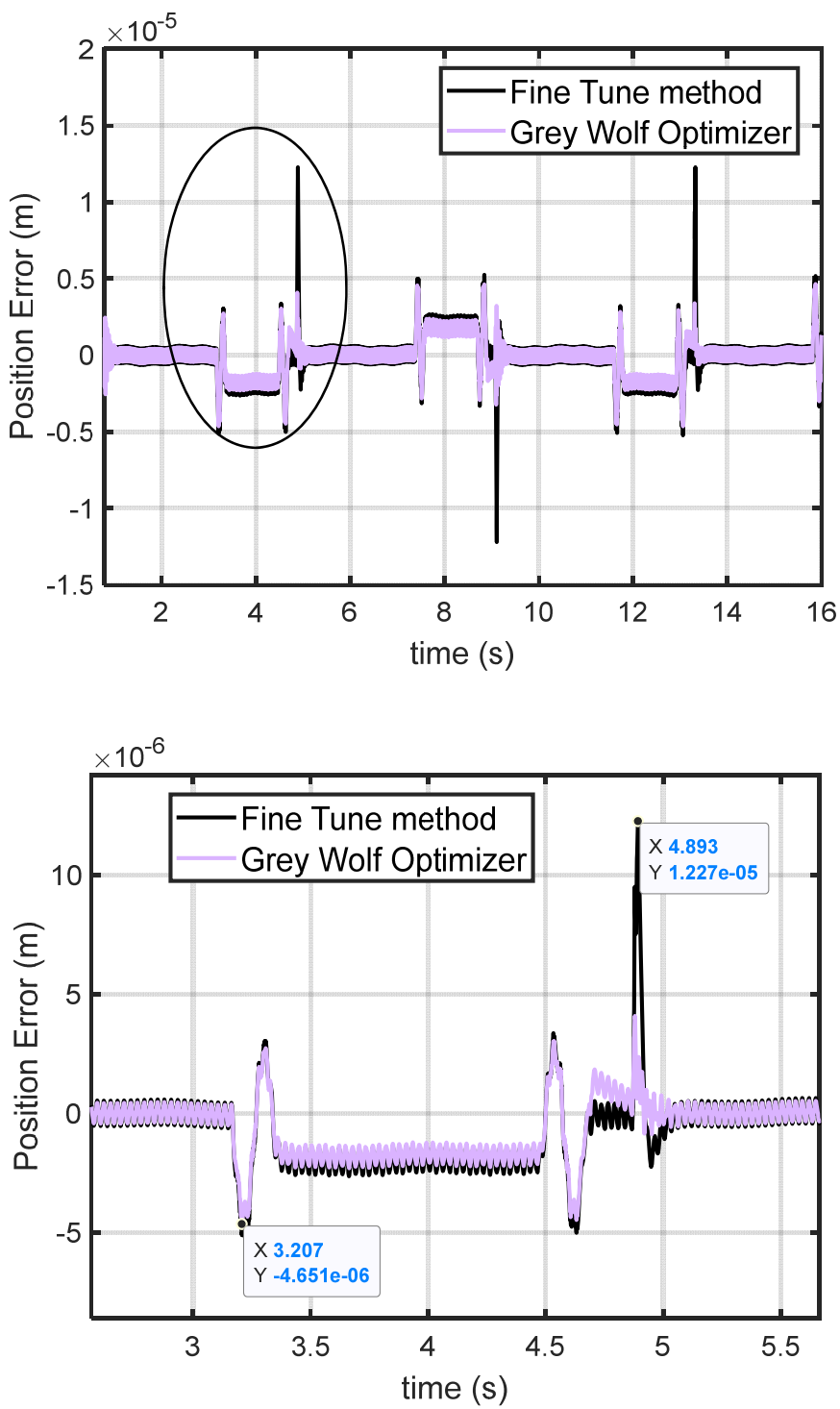

Fig. 5 Simulation results of FT and GWO for tuning cascade P-PI control system 


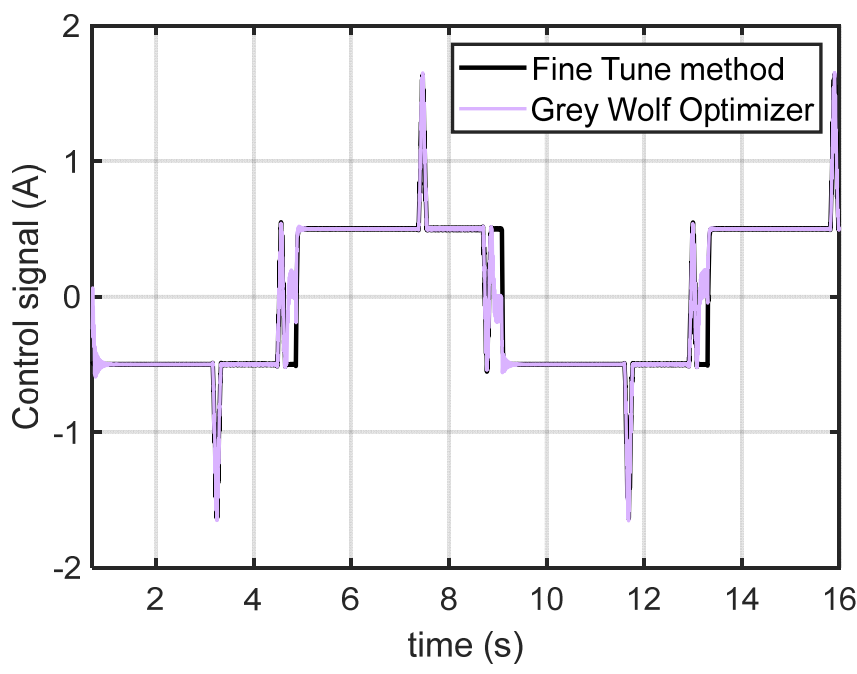

Fig. 6 Behavior of the simulated current signal for FT method and GWO algorithm

\section{B. Real-time experiments}

Real-time experiments for evaluating CNC machine tool positioning system with both set of parameters yielded by FT and GWO were conducted in the test platform shown in Fig. 2. The comparison of GWO and Fine Tuning method is carried out considering maxE, ITAE and IAU. Fig. 8 shows the resulting performance indices.

These experimental results are very well aligned with the simulation results, and corroborated that GWO outperforms Fine Tune Method by a remarkable reduction in the maxE with very good accuracy according to ITAE. This improvement can be quantified in $18 \%$ and $16 \%$ for maxE and ITAE, respectively. Moreover, the excellent behavior is also endorsed with a $2.42 \%$ reduction in the control effort. The obtained position error during the experiment is illustrated in Fig. 8 and the control signal is illustrated in Fig. 9 .

\section{- Fine Tune method $\quad$ Grey Wolf Optimizer}

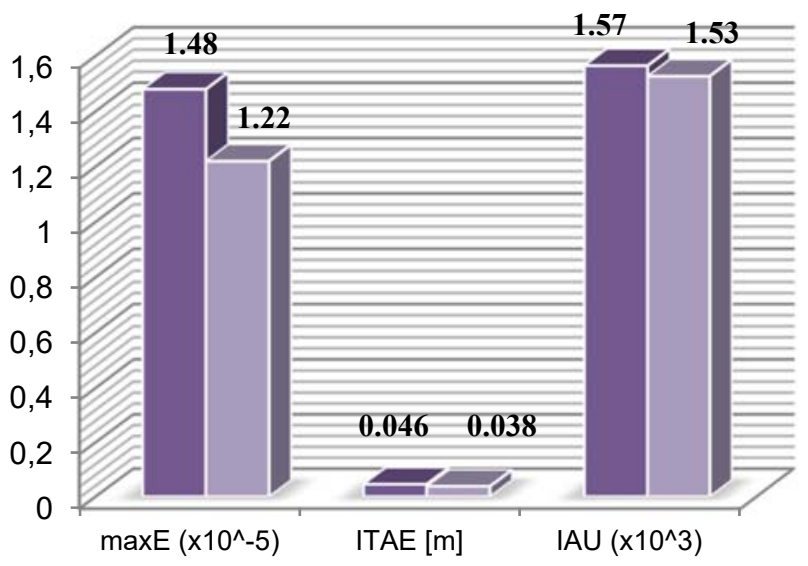

Fig. 7 Performance indices in experimental results

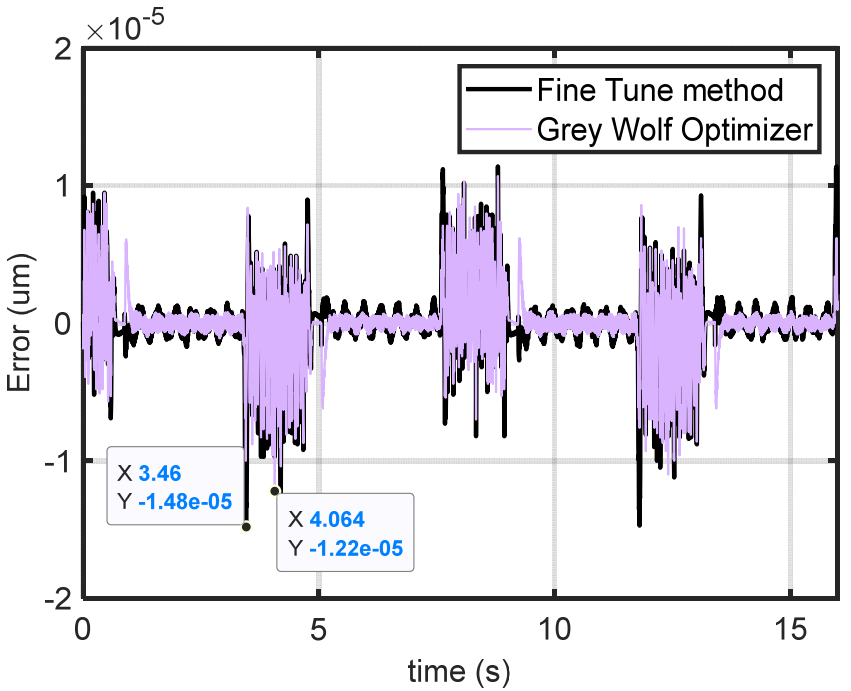

Fig. 8 Experimental results of position error when FT method and digital twin-based GWO method are applied.

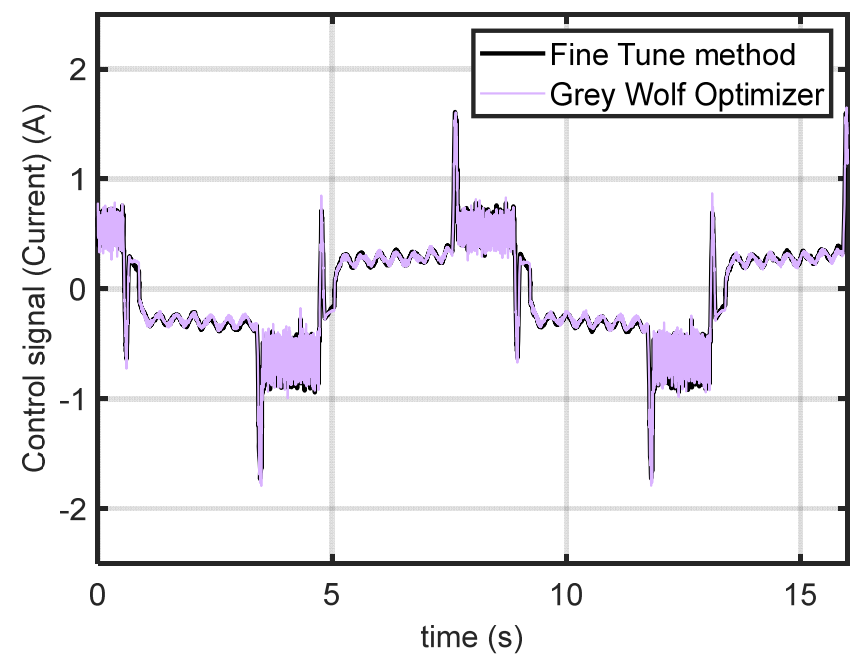

Fig. 9 Behavior of the control signal in both methods.

\section{CONCLUSION}

This paper proposes a digital twin-based optimization method supported on grey wolf optimizer algorithm for optimally adjusting parameters in ultraprecision motion systems widely applied in machine tool positioning systems. The objective function is to reduce as much as possible the maximum position error in presence of hard nonlinearities. This optimization process results in a set of six optimal parameters. Both simulation and experimental results are compared with industry driven method called Fine Tune (FT). The simulation results show a very remarkable improvement in accuracy and good transient response. The real-time experiments carried out on a testing platform with Fagor 8070 controllers also demonstrate the advantage of the GWO over FT method on the basis of lesser maximum peak error $(18 \%)$, better accuracy $(16 \%)$ with almost the same or less control effort $(2.42 \%)$.

\section{ACKNOWLEDGMENT}

The authors wish also to thank the NAWA Polish agency through the IPAE project: "Industry 4.0 in Production and Aeronautical Engineering". This work is also supported by H2020 project Grant 826417 Power2Power and DPI2017- 
86915-C3-1-R "Cognitive inspiration navigation for autonomous driving".

\section{REFERENCES}

[1] A. Villalonga, G. Beruvides, F. Castano, and R. Haber, "Industrial cyber-physical system for condition-based monitoring in manufacturing processes," in Proceedings - 2018 IEEE Industrial Cyber-Physical Systems, ICPS 2018, 2018, pp. 637642.

[2] B. R. Ferrer et al., "Towards the Adoption of Cyber-Physical Systems of Systems Paradigm in Smart Manufacturing Environments," in Proceedings - IEEE 16th International Conference on Industrial Informatics, INDIN 2018, 2018, pp. 792-799.

[3] S. Iarovyi, J. L. M. Lastra, R. Haber, and R. Del Toro, "From artificial cognitive systems and open architectures to cognitive manufacturing systems," in Proceeding - 2015 IEEE International Conference on Industrial Informatics, INDIN 2015, 2015, pp. 1225-1232.

[4] F. Castaño, S. Strzełczak, A. Villalonga, R. E. Haber, and J. Kossakowska, "Sensor reliability in cyber-physical systems using internet-of-things data: A review and case study," Remote Sensing, Article vol. 11, no. 19, 2019, Art. no. 2252.

[5] A. Villalonga, F. Castano, G. Beruvides, R. Haber, S. Strzelczak, and J. Kossakowska, "Visual analytics framework for condition monitoring in cyber-physical systems," in 2019 23rd International Conference on System Theory, Control and Computing, ICSTCC 2019 - Proceedings, 2019, pp. 55-60.

[6] R. H. Guerra, R. Quiza, A. Villalonga, J. Arenas, and F. Castano, "Digital Twin-Based Optimization for Ultraprecision Motion Systems with Backlash and Friction," IEEE Access, Article vol. 7, pp. 93462-93472, 2019, Art. no. 8759853.

[7] M. Macchi, I. Roda, E. Negri, and L. Fumagalli, "Exploring the role of Digital Twin for Asset Lifecycle Management," IFACPapersOnLine, Article vol. 51, no. 11, pp. 790-795, 2018.

[8] Q. Qi and F. Tao, "Digital Twin and Big Data Towards Smart Manufacturing and Industry 4.0: 360 Degree Comparison," IEEE Access, Article vol. 6, pp. 3585-3593, 2018.

[9] F. Tao, H. Zhang, A. Liu, and A. Y. C. Nee, "Digital Twin in Industry: State-of-the-Art," IEEE Transactions on Industrial Informatics, Article in Press 2018.

[10] F. Tao and M. Zhang, "Digital Twin Shop-Floor: A New ShopFloor Paradigm Towards Smart Manufacturing," IEEE Access, Article vol. 5, pp. 20418-20427, 2017, Art. no. 8049520.

[11] G. Beruvides, R. Quiza, R. Del Toro, and R. E. Haber, "Sensoring systems and signal analysis to monitor tool wear in microdrilling operations on a sintered tungsten-copper composite material," Sensors and Actuators, A: Physical, Article vol. 199, pp. 165175, 2013.

[12] R. M. Del Toro, M. C. Schmittdiel, R. E. Haber-Guerra, and R. Haber-Haber, "System identification of the high performance drilling process for network-based control," in 2007 Proceedings of the ASME International Design Engineering Technical Conferences and Computers and Information in Engineering Conference, DETC2007, 2008, vol. 1 PART B, pp. 827-834.

[13] Y. Cai, B. Starly, P. Cohen, and Y. S. Lee, "Sensor Data and Information Fusion to Construct Digital-twins Virtual Machine Tools for Cyber-physical Manufacturing," Procedia Manufacturing, Article vol. 10, pp. 1031-1042, 2017.

[14] C. Ganz, "Digital twin - Virtually identical?," ABB Review, Short Survey no. 2, pp. 94-95, 2018.

[15] R.-E. Precup and R.-C. David, Nature-Inspired Optimization Algorithms for Fuzzy Controlled Servo Systems. ButterworthHeinemann, 2019.

[16] R. E. Haber, G. Beruvides, R. Quiza, and A. Hernandez, "A simple multi-objective optimization based on the cross-entropy method," IEEE Access, Article vol. 5, pp. 22272-22281, 2017, Art. no. 8070310.

[17] G. Beruvides, R. Quiza, and R. E. Haber, "Multi-objective optimization based on an improved cross-entropy method. A case study of a micro-scale manufacturing process," Information Sciences, Article vol. 334-335, pp. 161-173, 2016.

[18] G. Beruvides, F. Castaño, R. E. Haber, R. Quiza, and A. Villalonga, "Coping with Complexity When Predicting Surface Roughness in Milling Processes: Hybrid Incremental Model with
Optimal Parametrization," Complexity, Article vol. 2017, 2017, Art. no. 7317254.

[19] F. Castaño, G. Beruvides, R. E. Haber, and A. Artuñedo, "Obstacle Recognition Based on Machine Learning for On-Chip LiDAR Sensors in a Cyber-Physical System," Sensors, vol. 17, no. 9, p. 2109, 2017.

[20] F. Castaño, G. Beruvides, A. Villalonga, and R. E. Haber, "Selftuning method for increased obstacle detection reliability based on internet of things LiDAR sensor models," Sensors (Switzerland), Article vol. 18, no. 5, 2018, Art. no. 1508.

[21] I. La Fe-Perdomo, G. Beruvides, R. Quiza, R. Haber, and M. Rivas, "Automatic Selection of Optimal Parameters Based on Simple Soft-Computing Methods: A Case Study of Micromilling Processes," IEEE Transactions on Industrial Informatics, Article vol. 15 , no. 2, pp. 800-811, 2019, Art. no. 8325494 .

[22] C. Cimino, E. Negri, and L. Fumagalli, "Review of digital twin applications in manufacturing," Computers in Industry, Article vol. 113, 2019, Art. no. 103130.

[23] E. Negri, L. Fumagalli, C. Cimino, and M. MacChi, "FMUsupported simulation for CPS digital twin," in Procedia Manufacturing, 2019, vol. 28, pp. 201-206.

[24] A. Gajate, R. E. Haber, J. R. Alique, and P. I. Vega, "Transductive-weighted neuro-fuzzy inference system for tool wear prediction in a turning process", In International Conference on Hybrid Artificial Intelligence Systems, pp. 113-120. Springer, Berlin, Heidelberg, 2009.

[25] R. E. Haber and J. R. Alique, "Nonlinear internal model control using neural networks: An application for machining processes," Neural Computing and Applications, Article vol. 13, no. 1, pp. 47-55, 2004.

[26] R. Haber-Guerra, S. Y. Liang, J. R. Alique, and R. Haber-Haber, "Fuzzy control of spindle torque in high-speed milling processes," J. Manuf. Sci. Eng. Trans. ASME, vol. 128, no. 4, pp. 1014-1018, 2006, doi: 10.1115/1.2194063.

[27] G. Beruvides, F. Castaño, R. Quiza, and R. E. Haber, "Surface roughness modeling and optimization of tungsten-copper alloys in micro-milling processes," Meas. J. Int. Meas. Confed., vol. 86, 2016, doi: 10.1016/j.measurement.2016.03.002

[28] Z. Sun, G. Pritschow, P. Zahn, and A. Lechler, "A novel cascade control principle for feed drives of machine tools," CIRP Annals, Article vol. 67, no. 1, pp. 389-392, 2018.

[29] A. L. Sangeetha, N. Bharathi, A. B. Ganesh, and T. K. Radhakrishnan, "Particle swarm optimization tuned cascade control system in an Internet of Things (IoT) environment," Measurement: Journal of the International Measurement Confederation, Article vol. 117, pp. 80-89, 2018.

[30] S. Mirjalili, Evolutionary algorithms and neural networks: Theory and applications (Studies in Computational Intelligence, no. 780). Cham (Switzerland): Springer, 2019.

[31] FagorAutomation, "CNC 8070. Users Manual," ed, 2016. 